\title{
Familial clustering of bicuspid aortic valve and its relationship with aortic dilation in first-degree relatives
}

\author{
Laura Galian-Gay, ${ }^{1}$ Amelia Carro Hevia, ${ }^{1}$ Gisela Teixido-Turà, ${ }^{1}$ \\ José Rodríguez Palomares, ${ }^{1}$ Laura Gutiérrez-Moreno, ${ }^{1}$ Giuliana Maldonado, ${ }^{1}$ \\ María Teresa Gonzàlez-Alujas, ${ }_{1}^{1}$ Augusto Sao-Aviles, ${ }_{1}^{1}$ Pastora Gallego, ${ }^{2}$ \\ Francisco Calvo-Iglesias, ${ }^{3}$ Javier Bermejo, ${ }^{4}$ Juan Robledo-Carmona ${ }^{5}$ Violeta Sánchez, ${ }^{6}$ \\ Daniel Saura, ${ }^{7}$ Teresa Sevilla, ${ }^{8}$ Sergio Burillo-Sanz, ${ }^{9}$ Andrea Guala, ${ }^{1}$ \\ David Garcia-Dorado, ${ }^{1}$ Arturo Evangelista, ${ }^{1}$ On behalf of the BICUSPID investigators
}

\begin{abstract}
- Additional material is published online only. To view please visit the journal online (http://dx.doi.org/10.1136/ heartjn-2018-313802).
\end{abstract}

For numbered affiliations see end of article.

\section{Correspondence to} Dr Arturo Evangelista, Department of Cardiology, Vall d'Hebron Hospital, Barcelona 08035, Spain; arturevangelista masip@gmail.com

Received 3 July 2018

Revised 30 August 2018 Accepted 6 September 2018 Published Online First 15 October 2018

\section{SLinked}

- http://dx.doi.org/10.1136/ heartjnl-2018-314063

Check for updates

(C) Author(s) (or their employer(s)) 2019. No commercial re-use. See rights and permissions. Published by BMJ.

To cite: Galian-Gay L, Carro Hevia A, Teixido-Turà G, et al. Heart 2019;105:603-608.

\section{ABSTRACT}

Objective Bicuspid aortic valve (BAV) is the most common congenital heart disease. This study aimed to determine the prevalence rate of BAV in first-degree relatives (FDR) and the inheritance pattern according to different morphotypes and aortic dilation.

Methods BAV probands were consecutively studied at eight tertiary referral centres. After sequential screening, FDR were included in the study. The BAV morphotype, aortic dilation and aortic phenotype were assessed by transthoracic echocardiography.

Results Seven hundred and twenty-four FDR of 256 BAV probands agreed to undergo family screening. The prevalence of BAV was $6.4 \%$ in FDR $(9.2 \%$ in men, $3.5 \%$ in women, $p=0.002$ ). Aortic dilation was diagnosed in $9.6 \%$ of FRD with tricuspid aortic valves (TAV), with a root phenotype in $2.7 \%$ and tubular in $6.9 \%$ and more frequently in the presence of arterial hypertension (OR 4.48; Cl 95\% 2.51 to 7.99; $p=0.0001$ ) and valvular regurgitation (OR 5.87, CI 95\% 1.37 to 25.16; $p=0.025)$. The heritability $\left(h^{2}\right)$ of BAV was highly significant $(0.47 ; p=0.002)$; however, no concordance was observed among valve morphotypes. Aortic dilation heritability was not significant.

Conclusions The BAV prevalence rate in FDR was low (6.4\%) but aortic dilation was observed in $9.6 \%$ of FDR with TAV. The heritability of BAV was high without concordance in valve morphotypes, and aortic dilation heritability was not observed. Patients with BAV should be made aware of its familial pattern.

\section{INTRODUCTION}

Although bicuspid aortic valve (BAV) is considered a relatively benign condition, significant aortic valve dysfunction, aortic dilation or aortic dissection can appear at any time in life. ${ }^{1-3}$ Several clinical and experimental studies confirmed the familial clustering of BAV. ${ }^{4-6}$ Some studies suggested an autosomal-dominant inheritance pattern, ${ }^{4-7}$ while others showed that although BAV heritability is almost entirely genetic, the inheritance pattern may be explained by a polygenic inheritance with incomplete penetrance and variable expression. ${ }^{5}$ Evidence exists that BAV results from abnormal fusion during embryonic development ${ }^{8}$; however, the specific initiating mechanisms are not fully understood. Different morphological types of BAV according to the cusps fused have been described, with the most frequent $(70 \%-80 \%)$ being right and left coronary cusp fusion with an anteroposterior opening configuration. $^{9}$ A pure BAV, without raphe, appears in $7 \%-15 \%$ of the population. ${ }^{11}$ Whether valve morphotype plays a role in the familial inheritance pattern remains unknown. Complications related to aortic valve dysfunction appear in $16 \%-68 \%{ }^{12}$ and aortic dilation in $20 \%-84 \%$ of adults with $\mathrm{BAV},{ }^{13}$ which may appear combined or alone. Aortic dilation has also been documented in first-degree relatives (FDR) of patients with tricuspid aortic valve (TAV). The prevalence of this phenomenon ranges from $3 \%{ }^{4}{ }^{10}$ to $32 \%{ }^{14}$; however, its inheritance pattern remains unknown, probably due to the different methods and heterogeneous nature of studies. BAV screening in FDR is considered appropriate in the current clinical practice guidelines; however, the degree of recommendation is not established. ${ }^{15} 16$ Data on the possible impact of screening on outcomes or cost-effectiveness are scant. ${ }^{17}$

The aims of this cross-sectional, multicentre study were: (1) to establish the prevalence rate of BAV among FDR using echocardiography; (2) to determine the inheritance pattern of BAV according to the different morphological types of valve, valve dysfunction and aortic dilation; and (3) to evaluate the presence and risk factors of aortic dilation in FDR.

\section{METHODS}

\section{Study population}

Patients with BAV documented by echocardiography were consecutively assessed prospectively at eight tertiary referral centres from 2012 to 2015. Exclusion criteria were a non-definitive diagnosis of BAV or the presence of another connective tissue disorder. From 852 patients included in our previous study, ${ }^{18} 256$ agreed to undergo family screening. Anamnesis, three-generation pedigree, 
physical examination and transthoracic echocardiography were performed in each index case. FDR (parents, siblings and children) were identified and invited to participate in the study. Those who finally agreed to be screened were included in the study. Approval of the hospital Ethics Committees was obtained for all participating centres. All participants provided their written informed consent.

\section{Clinical and family study}

Anthropometric measurements (height and weight) were taken and body mass index and body surface both calculated in all patients with BAV and their FDR. Clinical history, presence of cardiovascular risk factors (hypertension, diabetes, dyslipidaemia and smoking), comorbidities and other associated cardiac malformations were obtained by personal interview at the time of echocardiographic study and completed from medical records. Three generations per proband were included, yielding a median of 3 (IQR 2-4) family members per proband.

\section{Echocardiographic studies}

Transthoracic echocardiography was performed in all patients by experienced cardiologists using commercially-available, high-resolution ultrasound systems. Standard two-dimensional views were obtained and colour, continuous and pulsed Doppler were used to assess dysfunctional valve quantification according to European Association of Cardiovascular Imaging Standards. BAV was defined as the presence of two cusps and commissures, with or without partial or total raphe, in either structure. Each aortic valve was analysed and characterised in systole and diastole. Diagnosis was established by the systolic fish-mouth appearance of the orifice in parasternal short-axis views using a zoom tool.

According to the fusion pattern of commissures, three morphological types were considered: type R-L, fusion of the right and left cusps; type R-N, fusion of the right and non-coronary cusps; and type L-N, fusion of the left and non-coronary cusps.

Patients were categorised in three groups according to the degree and type of valvular dysfunction: a non-significant dysfunctional group, including normally functioning valve, mild aortic stenosis (AS) or mild aortic regurgitation (AR), an AS-dominant group and an AR-dominant group, when valve dysfunction was moderate or severe.
The aortic root and ascending aorta were assessed using the parasternal long-axis view and at least three measurements were taken (sinuses of Valsalva, sinotubular junction and tubular diameter), at end-diastole from leading edge to leading edge. ${ }^{16} \mathrm{~A}$ non-standard parasternal long-axis window with an upper interspace and medial transducer position was obtained to visualise distal ascending aorta diameter. We measured the maximum diameter that can be obtained in these views. Echocardiographic studies were centrally analysed by two expert ecchocardiographers (AC and GM) using dedicated software (Echo PAC, GE Healthcare, Little Chalfont, UK).

\section{Aortic dilation definition}

Recently, different studies showed aortic diameters to be strongly influenced by age, body size and sex. For this reason, the aorta is considered to be dilated when its diameter is $1.96 \mathrm{SD}$ above the predicted diameter for a particular patient. ${ }^{19}$ Thus, the Z-score was applied to all aortic measurements in our population. ${ }^{19}$ Dilation of the aorta was examined at aortic root (sinuses of Valsalva) and ascending aorta levels. The aorta phenotype classification was assigned according to the segment of the vessel showing the largest diameter. ${ }^{20}$

\section{Statistical analysis}

Quantitative variables are expressed as mean \pm SD and qualitative variables in percentages. Intergroup comparison was made using Student's $t$-test for continuous variables and Fisher's exact test for qualitative variables. When the distribution was not normal, the Mann-Whitney U test was used. The 95\% CI was calculated. Logistic regression was used to calculate the odds ratios and confidence intervals. A p value $<0.05$ was considered significant. Statistical test for intergroup comparisons where chosen under the assumption of independence between groups.

These analyses were made using the statistical software programme SPSS V.9.0 for Windows. Additive genetic heritability was calculated with the SOLAR genetic statistics package. This software calculates the fraction of the total variance that can be explained by additive genetic variance. The method compares the sporadic model and the polygenic model based on log-likelihood values. A subset of the whole cohort included in this work was used for heritability analysis. In order to have homogeneous pedigrees, only families with proband's siblings

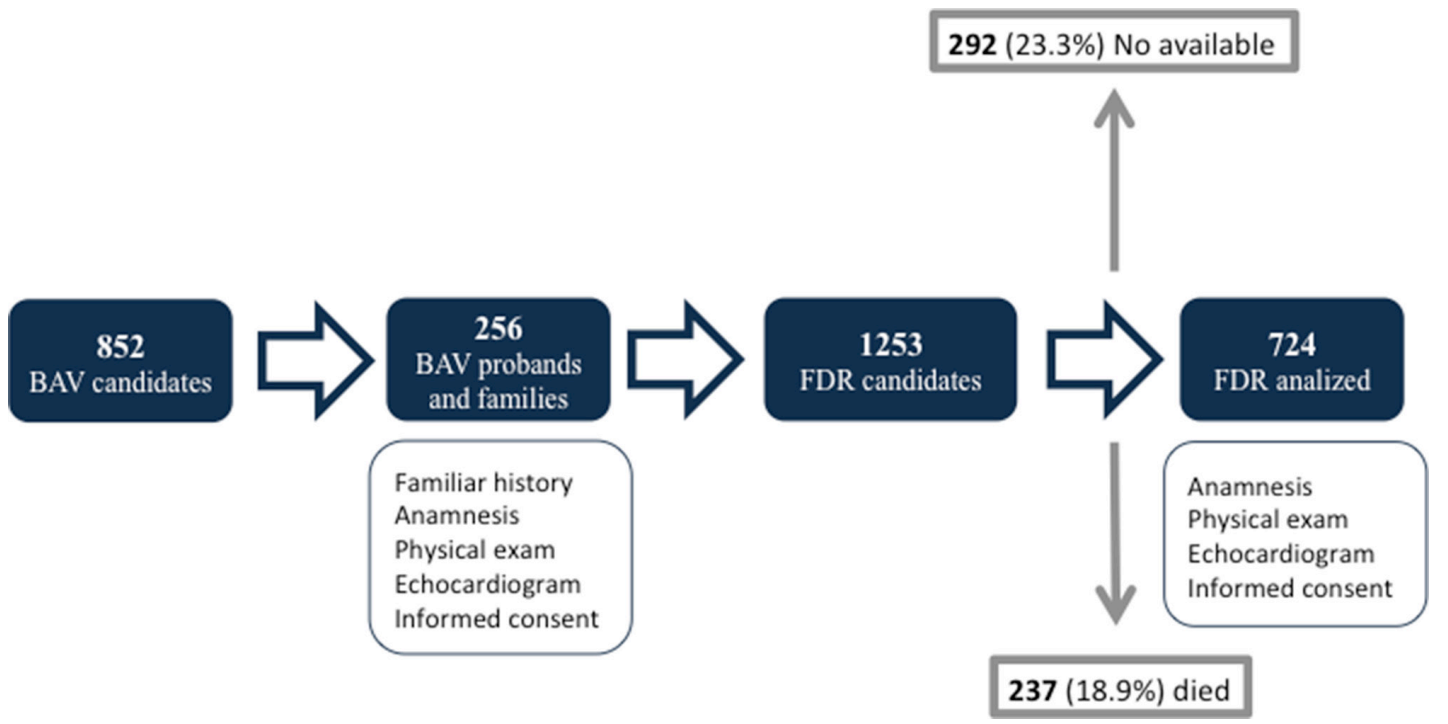

Figure 1 Representative scheme of study population. BAV, bicuspid aortic valve; FDR, first-degree relative. 
Table 1 Demographics, valve characteristics and aortic characteristics of all probands and FDRs

\begin{tabular}{|c|c|c|c|c|c|c|c|}
\hline & $\begin{array}{l}\text { All probands } \\
(n=256)\end{array}$ & $\begin{array}{l}\text { All FDRs } \\
(n=724)\end{array}$ & $\begin{array}{l}\text { P value } \\
\text { probands vs } \\
\text { BAV FDR }\end{array}$ & $\begin{array}{l}\text { FDRs with BAV } \\
(n=46)\end{array}$ & $\begin{array}{l}\text { FDRs without BAV } \\
(n=678)\end{array}$ & $\begin{array}{l}P \text {-values } \\
\text { BAV FDR vs } \\
\text { TAV FDR }\end{array}$ & $\begin{array}{l}\text { Statistical } \\
\text { power analysis } \\
\text { (1- } \beta \text { err prob) }\end{array}$ \\
\hline \multicolumn{8}{|l|}{ Demographic data } \\
\hline Age, years & $46.41 \pm 16.40$ & $41.68 \pm 18.66$ & 0.272 & $43.46 \pm 18.75$ & $41.55 \pm 18.66$ & 0.497 & 0.10 \\
\hline Males, N (\%) & $182(71)$ & $356(49.2)$ & 0.001 & $33(71.7)$ & $323(47.6)$ & 0.002 & 0.73 \\
\hline $\mathrm{BSA}, \mathrm{m}^{2}$ & $1.84 \pm 0.19$ & $1.73 \pm 0.29$ & 0.99 & $1.84 \pm 0.24$ & $1.72 \pm 0.29$ & 0.016 & 0.88 \\
\hline Hypertension, N (\%) & $84 / 227(37)$ & $101 / 718(14.1)$ & 0.001 & $12 / 43(27.9)$ & $89(13.2)$ & 0.012 & 0.73 \\
\hline Diabetes, N (\%) & $13 / 226(5.8)$ & - & & - & - & & \\
\hline Dyslipidaemia, N (\%) & $63 / 226(27.9)$ & - & & - & - & & \\
\hline Smoker, N (\%) & $73 / 225(32.4)$ & - & & - & - & & \\
\hline \multicolumn{8}{|l|}{ Valve characteristics } \\
\hline BAV R-L, N (\%) & $162 / 229(63.3)$ & - & & $34(73.9)$ & - & & \\
\hline BAV R-N, N (\%) & $60 / 229(26.2)$ & - & & $11(23.9)$ & - & & \\
\hline BAV L-N, N (\%) & $7 / 229(3.1)$ & - & & $1(2.2)$ & - & & \\
\hline Presence of raphe, $\mathrm{N}(\%)$ & $187 / 229(81.7)$ & - & & $34(73.9)$ & - & & \\
\hline \multicolumn{8}{|l|}{ Valve dysfunction } \\
\hline Non-dysfunction, N (\%) & $139(54.3)$ & 709 (97.9) & 0.001 & $40(87)$ & $669(98.7)$ & 0.001 & 0.93 \\
\hline AS-dominant, N (\%) & $50(19.5)$ & $3(0.4)$ & 0.01 & $2(4.3)$ & $1(0.1)$ & 0.001 & 0.99 \\
\hline AR-dominant, N (\%) & $67(26.2)$ & $12(1.7)$ & 0.01 & $4(8.7)$ & $8(1.2)$ & 0.001 & 0.81 \\
\hline \multicolumn{8}{|l|}{ Aortic diameters } \\
\hline Aortic root, mm & $36.61 \pm 5.75$ & $30.61 \pm 4.90$ & 0.024 & $34.54 \pm 5.45$ & $30.34 \pm 4.75$ & 0.001 & 0.99 \\
\hline Aortic root, $\mathrm{mm} / \mathrm{m}^{2}$ & $19.93 \pm 3.26$ & $17.98 \pm 4.12$ & 0.032 & $18.89 \pm 2.80$ & $17.91 \pm 4.19$ & 0.001 & 0.58 \\
\hline Aortic root, Z-score & $1.39 \pm 1.4$ & $1.05 \pm 0.22$ & 0.443 & $1.23 \pm 0.43$ & $1.04 \pm 0.2$ & 0.0001 & 0.82 \\
\hline Ascending tubular, mm & $40.83 \pm 7.72$ & $29.55 \pm 5.45$ & 0.001 & $35.24 \pm 7.41$ & $29.15 \pm 5.07$ & 0.001 & 0.99 \\
\hline Ascending tubular, $\mathrm{mm} / \mathrm{m}^{2}$ & $22.28 \pm 4.68$ & $17.33 \pm 4.30$ & 0.001 & $19.21 \pm 3.78$ & $17.20 \pm 4.31$ & 0.001 & 0.92 \\
\hline Ascending tubular, Z-score & $3.2 \pm 1.56$ & $1.12 \pm 0.32$ & 0.0001 & $1.47 \pm 0.5$ & $1.09 \pm 0.29$ & 0.0001 & 0.99 \\
\hline \multicolumn{8}{|l|}{ Aortic dilation } \\
\hline Dilation according to Z-score, N (\%) & $217(84.8)$ & $87(12)$ & 0.001 & $22(47.8)$ & $65(9.6)$ & 0.001 & 0.99 \\
\hline Dilation in the aortic root, $\mathrm{N}(\%)$ & $9(3.5)$ & $16(2.2)$ & 0.789 & $2(4.3)$ & $14(2.1)$ & 0.329 & 0.24 \\
\hline Dilation in ascending aorta, $\mathrm{N}(\%)$ & $208(81.3)$ & $71(9.8)$ & 0.001 & $20(43.5)$ & $51(7.5)$ & 0.001 & 0.99 \\
\hline Aortic root phenotype, N (\%) & $31(12.1)$ & $21(2.9)$ & 0.268 & $3(6.5)$ & $18(2.7)$ & 0.139 & 0.37 \\
\hline Ascending aorta phenotype, N (\%) & $186(72.7)$ & $66(9.1)$ & 0.001 & $19(41.3)$ & $47(6.9)$ & 0.001 & 0.99 \\
\hline Aortic dilation $\geq 45 \mathrm{~mm}, \mathrm{~N}(\%)$ & $85(33.2)$ & $6(0.8)$ & 0.0002 & $3(6.5)$ & $3(0.4)$ & 0.0001 & 0.82 \\
\hline Aortic dilation $\geq 50 \mathrm{~mm}, \mathrm{~N}(\%)$ & $31(12.1)$ & $3(0.4)$ & 0.268 & $3(6.5)$ & $0(0)$ & 0.0001 & - \\
\hline
\end{tabular}

In 27 cases, the valve morphology could not be definitively established.

$A R$, aortic regurgitation; $A S$, aortic stenosis; $B A V$, bicuspid aortic valve; $B S A$, body surface area; FDR, first-degree relative.

were included. The resulting subset was composed of 153 probands and 236 siblings. Heritability power was calculated with $h 2$ power command of SOLAR software with 10 replicates as the power to detect the corresponding observed heritability at a significance level of 0.05 .

\section{RESULTS}

Two hundred and fifty-six BAV probands from a BAV database agreed to undergo family screening. Through sequential screening techniques, 1253 FDR were identified as candidates for inclusion in the study. Of these, 237 (18.9\%) had died and $292(23.3 \%)$ were not available (refused to participate, changed residence or did not attend appointments). Finally, 724 FDR were included in the screening echocardiographic study (figure 1 ). The mean age of FDR was $41.7 \pm 18.7$ years and $49.2 \%$ were males. Forty-six (6.4\%) were identified as having a BAV, 218 probands had no relatives with BAV, 31 had one relative with BAV, 6 had two relatives with BAV and 1 had three relatives with BAV. A new affected member was identified in $14.8 \%$ of the families (46 BAV in FDR distributed in 38 families). FDRs with BAV were more frequently men $(71.7 \%$ vs $47.6 \% ; \mathrm{p}=0.002)$ and more often hypertensive (27.9\% vs $13.2 \% ; \mathrm{p}=0.012)$ (table 1$)$.

\section{Valve characteristics and dysfunction}

Characteristics and BAV morphotypes in probands are shown in table 1. BAV morphotypes among relatives were as follows: BAV R-L in 34 (73.9\%), BAV R-N in 11 (23.9\%) and BAV L-N in 1 (2.2\%). No significant relationship was found among probands and relatives according to BAV morphotype. Pure BAV without raphe was present in $26.1 \%$ of FDR with BAV with no significant differences with the probands. Regarding valve dysfunction, $45.7 \%$ of probands had dysfunctional aortic valves $(19.5 \%$ AS and $26.2 \%$ AR), while $13 \%$ of FDR with BAV had dysfunctional valves (4.3\% AS and $8.7 \%$ AR).

\section{Aortic dilation}

Aortic dilation was more frequent in FDR with BAV compared with FDR with TAV (47.8\% vs 9.6\%; p=0.001) (table 1). Dilation of the tubular ascending aorta was more frequent in FDR with BAV compared with FDR without BAV (20 (43.5\%) vs $51(7.5 \%) ; \mathrm{p}=0.001)$, while dilation of the aortic root was similar between groups $(2(4.3 \%)$ vs $14(2.1 \%)$; $p=0.329)$. The ascending aortic phenotype also appeared more frequently in the FDR with BAV group (19 (41.3\%) vs 47 (6.9\%); $\mathrm{p}=0.001)$, 
Table 2 Characteristics of FDRs with dilation and non-dilation of the aorta

\begin{tabular}{|c|c|c|c|c|}
\hline FDRs with TAV & $\begin{array}{l}\text { Non-dilated } \\
(n=613)\end{array}$ & $\begin{array}{l}\text { Dilated } \\
(n=65)\end{array}$ & $P$ values & $\begin{array}{l}\text { Statistical } \\
\text { power } \\
\text { analysis (1- } \beta \\
\text { err prob) }\end{array}$ \\
\hline Age, years & $40.7 \pm 18.4$ & $49.1 \pm 19.1$ & 0.0001 & 0.91 \\
\hline Males, N (\%) & 288/613 (47) & $35(53.8)$ & 0.299 & 0.18 \\
\hline Hypertension, N (\%) & $67 / 613(10.9)$ & $22 / 62(35.5)$ & 0.0001 & 0.99 \\
\hline \multicolumn{5}{|l|}{ Valve dysfunction } \\
\hline $\begin{array}{l}\text { Non-dysfunctional valve, } \\
\mathrm{N}(\%)\end{array}$ & $607 / 613(99)$ & $62 / 65(95.4)$ & 0.02 & 0.60 \\
\hline Presence of AS, N (\%) & $1 / 613(0.2)$ & $0 / 65(0)$ & 0.268 & - \\
\hline Presence of $A R, N(\%)$ & $5 / 613(0.8)$ & $3 / 65(4.6)$ & 0.006 & 0.16 \\
\hline
\end{tabular}

In three cases, hypertension variable could not be definitively established. AS, aortic stenosis; $A R$, aortic regurgitation; FDR, first-degree relative; TAV, tricuspid aortic valve.

but no significant differences were observed in the aortic root phenotype. A dilated aorta $\geq 45 \mathrm{~mm}$ was present in $6.5 \%$ of FDR with BAV and $0.4 \%$ of FDR with TAV and a significantly dilated aorta $(\geq 50 \mathrm{~mm})$ was present in $6.5 \%$ of FDR with BAV and $0 \%$ of FDR with TAV.

Sixty-five individuals $(9.6 \%)$ of the FDR with TAV had aortic dilation. They were older, with a higher prevalence of hypertension and AR, than those without dilation (table 2). History of hypertension and significant $\mathrm{AR}$ were more frequent in this population (OR 4.48; CI 95\% 2.51 to 7.99; $\mathrm{p}=0.0001$ and OR 5.87 , CI 95\% 1.37 to $25.16 ; \mathrm{p}=0.025$, respectively). Angio CT was performed in 22 FDR with aortic dilation from the coordinator centre. Mini-raphe (small fusion between cusps), not defined by TTE, was diagnosed in 9 of whom (figure 2), 5 of whom underwent 4D-MRI study which showed eccentric jet with an increase in rotational flow and axial wall shear stress.

\section{Heritability}

Significant heritability was found in our cohort of siblings for BAV $\left(b^{2}=0.47 ; \mathrm{p}<0.05\right)$ (table 3$)$. Overall aorta dilation heritability was not significant $(\mathrm{p}=0.06)$ and, since BAV and aortic dilation traits were associated (online supplementary table 1),

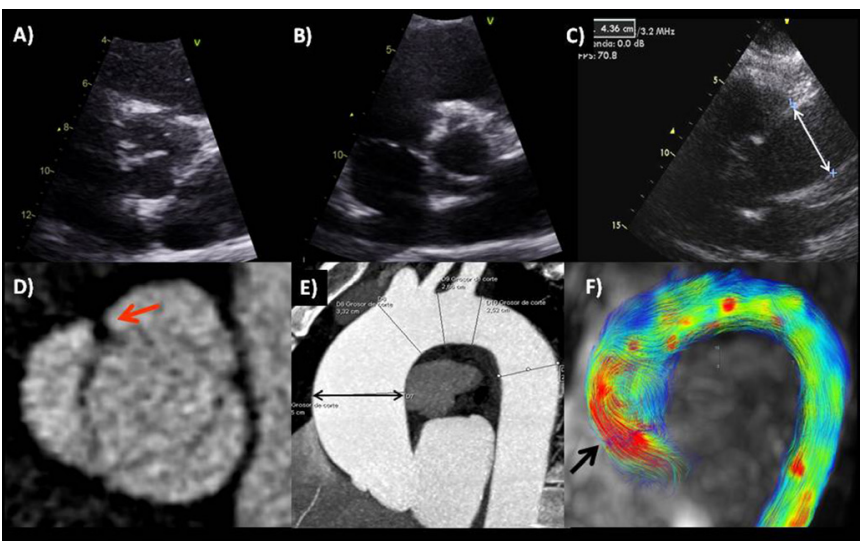

Figure 2 FDR of a patient with BAV showing a tricuspid leaflet valve in diastole (A) and systole (B) with ascending aortic dilation (C) in the bidimensional echocardiographic images. CT showed a mini-raphe (arrow) between left and right coronary cusps (D) and confirmed ascending aortic dilation (E). 4D-flow MRI revealed the eccentric direction of the aortic jet towards to the anterior wall of the ascending aorta (F). BAV, bicuspid aortic valve; FDR, first-degree relative. aortic dilatation $h^{2}$ was calculated in siblings without BAV and in siblings with BAV. Aortic dilation heritability did not reach statistical significance in any case.

Valvular morphotype in siblings with BAV $(n=43)$ was not associated with proband morphotype (online supplementary table 2), and the BAV prevalence rate was not related to proband BAV morphotype (online supplementary table 3 ).

\section{DISCUSSION}

This large multicentre study found the prevalence rate for FDR of BAV subjects to be $6.4 \%$, being higher in men $(9.2 \%)$ than in women $(3.5 \%)$. A new affected member was identified by conventional echocardiography in $14.8 \%$ of families. BAV heritability was moderate and not related to valve morphotype. Aortic dilation prevalence in FDR with TAV was relatively high (9.6\%). Most dilations were mild and more frequently observed in patients with hypertension. In one subgroup of patients with TAV with aorta dilation, CT defined the presence of a miniraphe not previously diagnosed by TTE (41\%).

This is the most extensive series to analyse the BAV prevalence rate in FDR. The characteristics of BAV probands in the current series were similar to those of other studies. BAV was more prevalent in men (71\% of cases), and BAV R-L more common than BAV-R-N (63.3\% vs $26.2 \%, \mathrm{p}<0.0001)$. Over $50 \%$ of our relatively young population (mean age: $46.4 \pm 16.4$ years) had no significant valvular dysfunction. The prevalence rate of BAV in FDR was $6.4 \%$, in the range of previously published data of $4.6 \%{ }^{10}$ to $11 \% .^{451421}$ Using a maximum-likelihood-based variance composition method, we estimated the heritability of BAV to be 0.47 , suggesting that in this population, the determination of $\mathrm{BAV}$ is not entirely genetic. Taken together, these results suggest that there may be a significant but incomplete genetic basis underlying BAV morphology. Some previous studies reported that heritability for BAV and other left ventricular outflow tract obstruction defects ranged from approximately 0.7 to 0.9 (5-6). These values were slightly higher than those reported in our series; however, we analysed isolated BAVs excluding other associated congenital heart defects, and the prevalence rate of the disease was significantly lower in comparison with other studies.

In contrast to the small series of Huntington $e t$ al in which the male-to-female ratio was $1: 1,{ }^{4}$ a similar ratio in probands and FDR with BAV was found in our larger study, which agrees with the ratio of community cohort studies. ${ }^{2}$

In our series, no significant relationship was found between probands and relatives according to BAV morphotype (56\% concordance). This finding concurs with the results of other series ${ }^{10}$ and supports the hypothesis that although BAV is determined by genetic factors, BAV morphology is defined in the embryological phase, and must be related to epigenetic and/or environmental factors. ${ }^{22}$

Dilation of the ascending aorta is common in patients with BAV and can occur in the absence of significant aortic valve dysfunction, providing support to the genetic/aortopathy theory. ${ }^{23}$ Some researchers suggest two different theories for the cause of aneurysms in patients with BAV (the haemodynamic theory vs the aortopathy theory). However, interaction between genetic substrate and haemodynamic factors and flow abnormalities is very likely to be responsible for the ultimate expression of the ascending aorta dilation. ${ }^{18} 24$

Results regarding a potential risk of thoracic aortic aneurysm in FDR with a TAV are controversial. Several authors have suggested that ascending aortic dilation is one component of the bicuspid syndrome and is inherited together with BAV, 
Table 3 Heritability

\begin{tabular}{lllll}
\hline & BAV & Dilation all siblings & Dilation in siblings without BAV & Dilation in siblings with BAV \\
\hline $\mathrm{N}$ & 389 & 389 & 371 & 18 \\
& $(153$ probands and 236 siblings) & $(153$ probands and 236 siblings) & (144 probands and 227 siblings) & (9 probands and nine siblings) \\
Prevalence in siblings (\%) & 9.3 & 14.8 & 13.9 & 50 \\
$\mathrm{~h}^{2}$ & 0.47 & 0.30 & 0.31 & 0.68 \\
$\mathrm{SE}$ & 0.18 & 0.21 & 0.23 & 0.94 \\
$\mathrm{P}$ value & 0.002 & 0.06 & 0.08 & 0.24 \\
$h^{2}$ power & $>0.9$ & $>0.9$ & $>0.9$ & $>0.9$ \\
\hline
\end{tabular}

BAV, bicuspid aortic valve; $h^{2}$, heritability; $h^{2}$ power, heritability power calculation; $N$, number of analysed individuals.

although both conditions do not necessarily appear together in the same individual. Some data suggest that the risk of thoracic aortic aneurysm is higher in relatives of patients with BAV even in those individuals who have morphologically normal aortic valves. Loscalzo et al found a $22 \%$ prevalence of aortic dilation in BAV families with or without BAV. ${ }^{7}$ Similarly, Biner et al reported a $32 \%$ prevalence of aortic root dilation in FDR, and also that aorta elastic properties were more affected in FDR than in controls but less than in BAV probands. ${ }^{14}$ However, in that study, $53 \%$ of patients with BAV index had a dilated aortic root. Dilation of the aortic root is described as relatively rare in $\mathrm{BAV}$ and is proposed as the form of bicuspid aortopathy most likely to be associated with a genetic cause. ${ }^{27132125-27}$ FDRs of young patients with BAV with aortic root dilation may have an increased risk of thoracic aortic aneurysm in absence of BAV. ${ }^{714}$ However, other studies reported lower percentages of 3\%-4\% of aorta dilation in first-degree TAV relatives ${ }^{4} 101721$ similar to the general population. Interestingly, Dayan et al compared a group of FDR of patients with BAV without aortic dilation with a group of FDR of patients with TAV without aortic dilation and found no differences in aortic measurements between groups, suggesting that aortic dilation in FDR should be assessed when the proband has aortic dilation. ${ }^{28}$

In the present study, aortic dilation in TAV relatives was 9.6\%, mostly in the tubular part. This higher incidence of aortic dilation may be due to a strict definition of aortic dilation using a Z-score-based criterion, mean FDR age of 42 years and 14\% of cases with arterial hypertension. However, aortic dilation was $\geq 45 \mathrm{~mm}$ in only $0.4 \%$ of FDR. In addition, aortic root dilation was significantly more infrequent than in the Biner et al's series $(2.1 \%$ vs $32 \%){ }^{14}$

Though not the aim of the current study, mini-raphe (frustre BAV) was identified by CT in 9 of 22 cases (41\%) with aortic dilation and apparent TAV by TTE. This interesting finding may widen our understanding of aortic dilation in FDR but should be interpreted with caution, as this was not the aim of our study. We hypothesise that these mini-raphes may disturb ascending aorta flow in a similar way to $\mathrm{BAV}^{24}$ (see figure 2). In a recent publication, surgical inspection of aortic valves in the setting of thoracic aneurysm and aortic insufficiency found very small raphes (ie, $3-6 \mathrm{~mm}$ in length) in valves initially considered to be tri-leaflet. These may have represented the fruste BAV form in 18/61 $(29.5 \%) .{ }^{29}$ Although these mini-raphes were not diagnosed by TTE, some were identifiable with high-resolution true co-axial imaging by TOE, CT or MRI. This fruste BAV form could play a role in the aortic dilation of FDR with TAV defined by TTE.

Although BAV is often identified incidentally in otherwise healthy, asymptomatic patients, it is associated with serious long-term health risks, including valvular dysfunction, infective endocarditis, significant aortic dilation and more exceptionally aortic. ${ }^{2}{ }^{30}$ Given its prevalence, and the fact that significant complications occur in over one-third of cases, BAV has a high morbidity rate. Based on these data, guidelines recommend echocardiographic evaluation of all FDR of patients with BAV as class IIaC in search of aortic dilation and/or BAV. ${ }^{16}$ Data of the current study need to be considered in these screening recommendations.

\section{Strengths and limitations}

This study was conducted in families of patients with BAV attending tertiary centres; thus, our population may have a selection bias. As described in other series, ${ }^{10} 17$ only a $30 \%$ of possible candidates agreed to undergo family screening, probably due to geographic reasons, lack of interest or considering BAV a benign abnormality. Pedigree was not obtained in all families and the genetic effect size (heritability) could not be estimated. The aim of the study was not to define mini-raphe prevalence. Therefore, only one subgroup of FDR with aortic dilation at the coordinator centre underwent CT study and this finding should be confirmed in further studies.

\section{CONCLUSIONS}

The BAV prevalence rate in FDR in this large multicentre study was $6.4 \%$, with no concordance in valvular morphotype. Aortic dilation was observed in $9.6 \%$ of FDR with TAV. The heritability of BAV was high without concordance in valve morphotypes but aortic dilation heritability was not observed. Patients with BAV

\section{Key messages}

What is already known on this subject?

- The prevalence of bicuspid aortic valve (BAV) in first-degree relatives (FDRs) varies from $4 \%$ to $11 \%$ in published series. While the presence of aortic dilation has also been documented, its inheritance pattern remains unknown.

\section{What might this study add?}

- The prevalence of BAV was $6.4 \%$ in FDR and aortic dilation appeared in $10 \%$ of FRD with tricuspid aortic valve, and this phenomenon was associated with arterial hypertension and aortic regurgitation. The heritability of BAV is moderate and suggests a mostly genetic pattern, which is also not subject to BAV morphotype. Aortic dilation heritability was not observed.

\section{How might this impact on clinical practice?}

- Aortic dilation is present not only in patients with BAV but also in their FDR. Patients with BAV should be made aware of its familial pattern. These data need to be considered in recommendations about screening. 
should be made aware of its familial pattern, and screening of their FDR advised. Further studies by CT or MRI are required to elucidate the presence and implications of mini-raphe in FDR with TAV and aortic dilation.

\section{Author affiliations}

'Department of Cardiology, Hospital Universitari Vall d'Hebron, CIBER-CV, Universitat Autònoma de, Barcelona, Spain

2Department of Cardiology, Hospital Universitario Virgen de la Macarena. CIBER-CV, Sevilla, Spain

${ }^{3}$ Department of Cardiology, Xestión Integrada de Vigo Hospital Alvaro Cunqueiro, Vigo, Spain

${ }^{4}$ Department of Cardiology, Hospital General Universitario Gregorio Marañón, Facultad de Medicina, Universidad Complutense de Madrid and CIBER-CV, Madrid, Spain

${ }^{5}$ Department of Cardiology, Hospital Universitario Virgen de la Victoria, CIBER-CV, Málaga, Spain

${ }^{6}$ Department of Cardiology, Hospital Universitario 12 de Octubre, CIBER-CV, Madrid, Spain

Department of Cardiology, Hospital Clínico Universitario Virgen de la Arrixaca, CIBER-CV, Murcia, Spain

${ }^{8}$ Department of Cardiology, Hospital Clínico Universitario de Valladolid, CIBER-CV, Valladolid, Spain

${ }^{9}$ Department of Immunology, Hospital Universitario Virgen del Rocío (IBiS, CSIC, US), Sevilla, Spain

Acknowledgements The authors would like to thank Christine O'Hara for help with the English language of the manuscript and Augusto Cesar Sao-Avilés for statistical analyses.

Collaborators BICUSPID investigators (see online supplementary appendix). This research was possible thanks to the collaboration of patients with BAV, their families and the staff of the following centres: Hospital Universitari Vall d'Hebron (Barcelona), Hospital Universitario Virgen de la Macarena (Sevilla), Hospital Universitario Virgen de la Victoria (Málaga), Hospital Alvaro Cunqueiro (Vigo), Hospital General Universitario Gregorio Marañón (Madrid), Hospital Clínico Universitario Virgen de la Arrixaca (Murcia), Hospital Univesitario 12 de Octubre (Madrid) and Hospital Clínico Universitario de Valladolid (Valladolid).

Contributors All co-authors and collaborators agree with the final version of the manuscript submitted and in the information that I have included in the current form.

Funding This work was supported by the grant PI11/01081 (Instituto de Salud Carlos III, Madrid).

Competing interests None declared.

Patient consent Obtained.

Ethics approval CEIC Hospital Vall d'Hebron.

Provenance and peer review Not commissioned; externally peer reviewed.

\section{REFERENCES}

1 Roberts WC. The congenitally bicuspid aortic valve. A study of 85 autopsy cases. Am J Cardiol 1970;26:72-83.

2 Michelena HI, Desjardins VA, Avierinos JF, et al. Natural history of asymptomatic patients with normally functioning or minimally dysfunctional bicuspid aortic valve in the community. Circulation 2008;117:2776-84.

3 Tzemos N, Therrien J, Yip J, et al. Outcomes in adults with bicuspid aortic valves. JAMA 2008; $300: 1317-25$.

4 Huntington K, Hunter AG, Chan KL. A prospective study to assess the frequency of familial clustering of congenital bicuspid aortic valve. J Am Coll Cardiol 1997;30:1809-12.
5 Cripe L, Andelfinger G, Martin LJ, et al. Bicuspid aortic valve is heritable. J Am Coll Cardiol 2004:44:138-43.

6 Calloway TJ, Martin LJ, Zhang X, et al. Risk factors for aortic valve disease in bicuspid aortic valve: a family-based study. Am J Med Genet A 2011;155A:1015-20.

7 Loscalzo ML, Goh DL, Loeys B, et al. Familial thoracic aortic dilation and bicommissural aortic valve: a prospective analysis of natural history and inheritance. Am J Med Genet A 2007;143A:1960-7.

8 Padang R, Bagnall RD, Semsarian C. Genetic basis of familial valvular heart disease. Circ Cardiovasc Genet 2012;5:569-80.

9 Bm S, Lewin MB, Stout KK, et al Heart 2008:94:1634-8.

10 Robledo-Carmona J, Rodríguez-Bailón I, Carrasco-Chinchilla F, et al. Hereditary patterns of bicuspid aortic valve in a hundred families. Int I Cardiol 2013;168:3443-9.

11 Sievers HH, Schmidtke C. A classification system for the bicuspid aortic valve from 304 surgical specimens. J Thorac Cardiovasc Surg 2007;133:1226-33.

12 Masri A, Svensson LG, Griffin BP, et al. Contemporary natural history of bicuspid aortic valve disease: a systematic review. Heart 2017;103:1323-30.

13 Verma S, Siu SC. Aortic dilatation in patients with bicuspid aortic valve. N Eng/ J Med 2014:370:1920-9

14 Biner S, Rafique AM, Ray I, et al. Aortopathy is prevalent in relatives of bicuspid aortic valve patients. J Am Coll Cardiol 2009:53:2288-95.

15 Vahanian A, Alfieri O, Andreotti F, et al. Guidelines on the management of valvular heart disease (version 2012). Eur Heart J 2012:33:2451-96.

16 Erbel R, Aboyans V, Boileau C, et al. ESC guidelines on the diagnosis and treatment of aortic diseases. Eur Heart J 2014 35:2873-926.

17 Panayotova R, Macnab A, Waterworth PD. A pilot project of familial screening in patients with bicuspid aortic valve disease. J Heart Valve Dis 2013;22:150-5.

18 Evangelista A, Gallego P, Calvo-Iglesias F, et al. Anatomical and clinical predictors of valve dysfunction and aortic dilation in bicuspid aortic valve disease. Heart 2018:104:566-73.

19 Campens L, Demulier L, De Groote K, et al. Reference values for echocardiographic assessment of the diameter of the aortic root and ascending aorta spanning all age categories. Am J Cardiol 2014;114:914-20.

20 Della Corte $\mathrm{A}$, Bancone $\mathrm{C}$, Buonocore $\mathrm{M}$, et al. Pattern of ascending aortic dimensions predicts the growth rate of the aorta in patients with bicuspid aortic valve. JACC Cardiovasc Imaging 2013;6:1301-10.

21 Martin LJ, Hinton RB, Zhang X, et al. Aorta Measurements are Heritable and Influenced by Bicuspid Aortic Valve. Front Genet 2011;2.

22 Fernández B, Durán AC, Fernández MC, et al. Genetic contribution of bicuspid aortic valve morphology. Am J Med Genet A 2011;155A:2897-8.

23 Keane MG, Wiegers SE, Plappert T, et al. Bicuspid aortic valves are associated with aortic dilatation out of proportion to coexistent valvular lesions. Circulation 2000;102:III-35--30

24 Rodríguez-Palomares JF, Dux-Santoy L, Guala A, et al. Aortic flow patterns and wall shear stress maps by 4D-flow cardiovascular magnetic resonance in the assessment of aortic dilatation in bicuspid aortic valve disease. J Cardiovasc Magn Reson 2018;20:28.

25 Girdauskas E, Rouman M, Disha K, et al. Aortopathy in patients with bicuspid aortic valve stenosis: role of aortic root functional parameters. Eur J Cardiothorac Surg 2016;49:635-44

26 Tadros TM, Klein MD, Shapira OM. Ascending aortic dilatation associated with bicuspid aortic valve: pathophysiology, molecular biology, and clinical implications. Circulation 2009:119:880-90.

27 Della Corte A, Bancone C, Quarto C, et al. Predictors of ascending aortic dilatation with bicuspid aortic valve: a wide spectrum of disease expression. Eur J Cardiothorac Surg 2007:31:397-405.

28 Dayan V, Parma G, Drever M, et al. First-degree relatives of bicuspid aortic valve patients with normal aortic dimensions do not carry an increased risk of aortic dilatation. Int J Cardiol 2014;172:518-9.

29 Sperling JS, Lubat E. Forme fruste or 'Incomplete' bicuspid aortic valves with very small raphes: The prevalence of bicuspid valve and its significance may be underestimated. Int I Cardiol 2015:184:1-5.

30 Lewin MB, Otto CM. The bicuspid aortic valve: adverse outcomes from infancy to old age. Circulation 2005;111:832-4. 